ORIGINAL ARTICLE

\title{
Iron Deficiency Association as A Possible Cause of First Febrile Seizure
}

\author{
ZARMAST KHAN ${ }^{1}$, ZABIT KHAN NAIBZAI ${ }^{2}$, MUHAMMAD ADNAN AHMED SETHI $^{3}$, SHAHID GHAFFAR ${ }^{4}$, SUMERA \\ MAKHDOOM ${ }^{5}$, ASADULLAH KHAN ${ }^{6}$ \\ ${ }^{1}$ Assistant Professor Pediatrics, Shifa College of Medicine, Islamabad \\ ${ }^{2}$ Sheikh Zayed University, Medical faculty, Pediatric department, KhostAfghanistan \\ ${ }^{3}$ Consultant Pediatrician, Paediatric Department, Tehsil Headquarter Hospital, Gujjar Khan \\ ${ }^{4}$ Assistant Professor, Department of Pediatrics Hamdard College of Medicine and Dentistry, Karachi \\ ${ }^{5}$ MBBS, (FCPS) Peads Medicine, WMO Paedriatic department, Liyari General Hospital, Karachi \\ ${ }^{6}$ Specialist PaediatricianAlkhidmat Hospital, Peshawar \\ Corresponding author:Dr. Zarmast Khan, Email Address: drzarmastkhan@ymail.com, Cell No. +923425408123
}

\begin{abstract}
Aim: A controlled clinical study was conducted to investigate the relationship between iron deficiency and first episode of febrile seizure in children 6-60 months of age.

Place and Duration:In the Pediatric Medicine department of ShifalnternationalHospital Islamabadforsix months duration from $15^{\text {th }}$ May 2020 to $30^{\text {th }}$ November 2020.

Methods:Hematological parameters for iron levels, including HB, MCV, MCHC and plasma ferritin, were determined in 75 patients who experienced a first febrile episode. 75 children without febrile seizures were taken as controls and the same markers were compared in both groups.

Results: The most important factors influencing the first attack of febrile seizure were HB, MCV, and serum ferritin levels.

Conclusion: Iron deficiency is significantly associated with the occurrence of the first febrile episode in children. Key words:Iron deficiency anemia, Iron deficiency, Febrile convulsions, Febrile attacks.
\end{abstract}

\section{INTRODUCTION}

Iron deficiency anemia is common in children in third world countries ${ }^{1}$. Almost one in three children suffers from iron deficiency (ID) due to poverty, lack of iron-rich foods, malabsorption or worm infestation. Iron is an essential micronutrient for growth, but it also plays a role in various brain processes such as neuro-metabolism, myelination and the action of neurotransmitters ${ }^{2-3}$. In patients with iron deficiency, the metabolism of monoamines and aldehyde oxidases is lowered, so the threshold for iron deficiency children is low and the risk of febrile seizures (FS) increases ${ }^{4-5}$. The type of iron deficiency may affect duration and recurrence of seizures ${ }^{6-7}$. FS is the most common form of childhood seizures ${ }^{8}$. Recent efforts have been made to find appropriate risk factors. FS has been extensively studied over the past two decades, and risk factors such as high fever, family history, and number of episodes per year are well documented. The link between ID and FS has been established in Kenya, an endemic region of malaria ${ }^{9}$. This study was conducted to further explore the relationship between ID and FS in our population where all risk factors are present. Although ID can usually be properly corrected, FS is an alarming experience for the family and there is a risk of caries. Therefore, as little work was done in this area, it was believed that research was needed to define the relationship between the two dominant states in Pakistan ${ }^{10-11}$. The aim of the study was to determine the relationship between iron deficiency and the first attack of febrile seizurein children admitted to the emergency department of Shifa International Hospital Islamabad.

\section{PATIENTS AND METHOD}

This case-control clinical trial was held at Pediatric Medicine department of Shifalnternational Hospital
Islamabad for six months duration from $15^{\text {th }}$ May 2020 to $30^{\text {th }}$ November 2020.

Inclusion Criteria: All children 6 months to 5 years of age who had their first febrile seizure attack in six months were enrolled in the study. First febrile attack (FFS) was defined as a febrile attack in children 6 months to 5 years of age, but there were no signs of central nervous system (CNS) infection or other definitive cause of the seizure.

Exclusion criteria: Children with a history of developmental delays, CNS infections, or neurological deficits were excluded from the study group.

Methods: Seizure onset, duration and family history were recorded only after the patient's condition had stabilized. Hematology tests including hemoglobin (HB), mean blood cell volume (MCV), mean corpuscular hemoglobin concentration (MCHC), and plasma ferritin (PF) were performed on the same blood sample.

A total of 75 patients who reported to the emergency department participated in the study were taken as studied group. A reference group of 75 patients was selected from hospitalized children for febrile conditions without seizures and admitted for such as diarrhea, respiratory diseases, otitis media, etc. were taken as control group. Written informed consent was obtained from parents of both groups. As stated above, samples were also taken from this group for hematology tests.

Statistical Analysis: Data were analyzed by a SPSS V-21 (SPSS-Inc., Chicago, USA). The frequency distribution was created by grouping the age of the subjects. Median \pm IQR was used to express the data. A logistic regression model with statistical significance $p$ $<0.05$ was used to determine a significant predictor of FFS.

\section{RESULTS}

The overall mean age of the patients and controls was $15.32 \pm 8.49$ and $15.60 \pm 9.51$ months; The maximum 
number of cases decreased in the 6-12-month group (Fig. 1). The mean hematology values ( $\mathrm{HB}, \mathrm{MCV}, \mathrm{MCHC}$, and PF) of the cases were found to be lower than that of the control group, as shown in Table 1.

A Comparison of median values of hematological variables of iron deficiency betweencontrols and cases shown in Table-I

\begin{tabular}{|l|c|l|}
\hline \multirow{2}{*}{ Variable } & Case & \multicolumn{1}{|c|}{ Control } \\
\cline { 2 - 3 } & Median $\pm \mathrm{IQR}$ & Median $\pm \mathrm{IQR}$ \\
\hline $\mathrm{MCHC}$ & $29.00 \pm 1.50$ & $33.00 \pm 3.50$ \\
\hline $\mathrm{HB}$ & $8.40 \pm 1.78$ & $9.00 \pm 2.28$ \\
\hline MCV & $69.00 \pm 1.50$ & $75.00 \pm 3.50$ \\
\hline Ferritin & $19.13 \pm 1.68$ & $24.02 \pm 2.23$ \\
\hline
\end{tabular}

HB levels were below $10 \mathrm{~g} / \mathrm{dL}$ in 52 people (69.33\%) compared to the control group (28.0\%). Similarly, the number of cases with hematological variables below normal was higher among the cases (Table 2).

Percentage of cases / controls with low level ofMCV, HB, PF and $\mathrm{MCHC}$ given in Table-II

\begin{tabular}{|l|l|l|}
\hline Variable & $\begin{array}{l}\text { Cases Number } \\
(\%)\end{array}$ & $\begin{array}{l}\text { Controls } \\
\text { Number (\%) }\end{array}$ \\
\hline $\mathrm{HB}<10 \mathrm{Gm} . / \mathrm{dl}$ & $52(69.33)$ & $21(28.0)$ \\
\hline $\mathrm{MCV}<72 \mathrm{fL}$ & $51(68.00)$ & $14(18.67)$ \\
\hline $\mathrm{MCHC}<32 \mathrm{Gm} . / \mathrm{dl}$ & $59(78.67)$ & $13(17.33)$ \\
\hline $\mathrm{PF}<20 \mathrm{ug} / \mathrm{L}$ & $37(49.33)$ & $29(38.67)$ \\
\hline
\end{tabular}

The most statistically significant factors affecting FFS are HB level, MCV level and PF level (Table 3).

Logistic Regression Model shown in Table-III

\begin{tabular}{|l|l|l|l|}
\hline Variables & $\begin{array}{l}\text { Regression } \\
\text { coefficient }\end{array}$ & Valid statistic & Significance \\
\cline { 4 - 4 } & -0.498 & 4.428 & \\
\hline Hemoglobin & -0.579 & 10.726 & 0.025 \\
\hline MCHC & -0.291 & 1.697 & 0.001 \\
\hline Ferritin & 0.07 & 12.44 & 0.181 \\
\hline
\end{tabular}

\section{DISCUSSION}

Iron deficiency is one of the most common micronutrient deficiencies in children in the developing world ${ }^{9-10}$. Even after anemia and iron deficiency have been corrected, it is strongly associated with persistent cognitive and motor delays ${ }^{11}$. There is controversy over the role of "iron status" in RCTs. Pisacane et al reported a significantly higher rate of iron deficiency anemia in children with FFS compared to the control group in a case-control study of 146 cases over a period of 6-24 months and 293 in the control group; Naveed-ur-Rehman and Billo proposed the same result in a case-control study of 30 cases and 30 controls ${ }^{11-12}$. Daoud et al. In a case-control study of 75 cases and 75 controls, with no significant difference in $\mathrm{HB}, \mathrm{MCV}$, and $\mathrm{MCH}$, only low PF levels were associated with FFS and could play a role; In contrast, Kobrinsky et al showed in a case-control study of 25 cases and 26 controls that anemia raises the FFS threshold, and iron deficiency may protect against the development of febrile seizures ${ }^{13-14}$. FFS was more common in the 6-12-month age group than in the 12-24month age group. Weaning practices are weak as most of our patients and controls are in the lower middle of the socioeconomic class. Other hematological markers of ID other than significantly low levels of PF and MCHC are consistent with the results of similar field studies ${ }^{15-16}$. Acute phase reagent PF may be falsely elevated in cases, which is a flaw in our study. Accompanying C-reactive protein can identify this tendency. However, despite the erroneous assumption of high infection rates, the number of cases with low ferritin levels is even higher than in the control group ${ }^{17-18}$. Moreover, the controls were obtained from the same environment and are equally likely to produce such results. Zareifar et al. Contrary to a recent study by 26 people, our history shows lower average blood volume as well as lower levels of HB and PF in the cases ${ }^{19-20}$.

\section{CONCLUSION}

The role of iron deficiency as a risk factor for febrile seizures is controversial. Some studies show that this is significantly correlated, while others have been unable to identify any correlation. While our study clearly shows a causal link between iron deficiency and the first bout of febrile seizure, keep in mind that in developing countries such as Pakistan, iron deficiency is much more common in hospitalized children than in developed countries. Therefore, large population studies are needed to investigate the coexistence of two common diseases.

\section{REFERENCES}

1. Jang, Han $\mathrm{Na}$, Hoi Soo Yoon, and EunHye Lee. "Prospective case control study of iron deficiency and the risk of febrile seizures in children in South Korea." BMC pediatrics 19, no. 1 (2019): 1-8.

2. Birua, Shyama, Suman Sarkar, AparajitaBera, and Kalimuddin Khan. "Clinico-Demographic Profile of Febrile Seizure and Its Association With Iron Deficiency." Journal of Nepal Paediatric Society 39, no. 2 (2019): 72-78.

3. Shaheed, Ahmed H., and Sawsan S. Abbas. "The Association Between Iron Deficiency and Febrile Seizures in Children Below 5 Years." Iraqi Journal of Medical Sciences 18, no. 2 (2020).

4. Yarigarravesh, Mohammad Hadi, Shadilzadbakhsh, ParastooAmiri, Maryam Goudarzian, Mehdi JaliliAkbariyan, and KazemHassanpour. "The Relationship between Iron Deficiency Anemia and Simple Febrile Convulsion in Children Aged 6 Months to 5 years." European Journal of Molecular \& Clinical Medicine 8, no. 3 (2021): 1049-1057.

5. Kumar, Navneet, TanuMidha, and Yashwant Kumar Rao. "Risk factors of recurrence of febrile seizures in children in a tertiary care hospital in Kanpur: A one year follow up study." Annals of Indian Academy of Neurology 22, no. 1 (2019): 31.

6. Shaheed, A. H., and S. A. Abbas. "The association between iron deficiency and febrile seizures in children below 5 years. Iraqi JMS. 2020; 18 (2): 110-116. doi: 10.22578." IJMS 18, no. 4.

7. Vaghela, Radhika, and Sanjay Mandot. "A case-control study to find the association between iron deficiency anemia and simple febrile seizures in children between 6 months and 5 years of age group." Indian Journal of Child Health 7, no. 2 (2020): 57-59.

8. Khanna, Alok, Ashok Kumar, DayanandHota, Aarti Sharma, and Manish Swami. "Relationship between iron deficiency anemia and febrile seizures: A case-control study." Medical Journal of Babylon 17, no. 2 (2020): 144.

9. Noorbakhsh, Samileh. "Association Between Iron Deficiency and Febrile Seizure? A Case Control Study in Tehran, Iran." (2021).

10. KHOSRAVI, SHAQAYEQ, SAYED MOHAMMAD HADI SADATI, VAHIDEH AL_SADAT SHERAFAT, and ZAHRA BAZARGANI. "Evaluation of Diagnostic Value of Blood 
Indices associated with Microcytic Anemia in Febrile Seizures in children."

11. Ehsanipour, Fahimeh, SamilehNoorbakhsh, AzitaTavasooli, and Leila Tahernia. "Association between iron deficiency and provoked febrile seizure in a middle income country: A case control study in Tehran, Iran." (2020).

12. SUJATHA, BALAMMA, KISHORE NARAYAN, LAL DEVAIYANAI VASUDEVAN NAIR, and HARSHITHA SHANMUGANATHAN. "Impact of Nutritional Status and Vitamin D Levels in Children with Febrile Seizure from a Suburban Tertiary Hospital in Tamil Nadu, India." Journal of Clinical \& Diagnostic Research 14, no. 9 (2020).

13. Abdelrahman, Rana A., Mohamed A. Shokeir, and Dina S. AbdElmagid. "Serum zinc level in children presenting with febrile seizures." Alexandria Journal of Pediatrics 33, no. 3 (2020): 144.

14. Tripathy, Debajyoti, Rampal Singh Tomar, Ashish Kumar Gupta, and RanjitGhuliani. "The risk associated with iron deficiency anemia for simple febrile seizures in children: A case-control study." Indian Journal of Child Health 7, no. 1 (2020): 25-28.

15. Aliabadi, GhasemMiri, Ali Khajeh, AlirezaOveisi, and MahsaPoorjangi. "Prevalence of Febrile Seizures in Children in Zahedan, South East of Iran." Iranian journal of child neurology 13, no. 3 (2019): 93.
16. Mantadakis, Elpis, EleftheriosChatzimichael, and PanagiotaZikidou. "Iron deficiency anemia in children residing in high and low-income countries: risk factors, prevention, diagnosis and therapy." Mediterranean Journal of Hematology and Infectious Diseases 12, no. 1 (2020).

17. Mhaibes, Sarah Hashim, Mustafa Shihab Al-Anbaki, and EmanSaadi Saleh. "The role of electrolytes and inflammatory biomarkers in the development of febrile seizure in children." Annals of Tropical Medicine and Health 23 (2020): 231-365.

18. Heydarian, Farhad, AlirezaAtaeiNakhaei, HasanMehradMajd, and ElhamBakhtiari. "Zinc deficiency and febrile seizure: a systematic review and metaanalysis." Turkish Journal of Pediatrics 62, no. 3 (2020).

19. Chan, Avis, Hannah Karpel, Ellen Spartz, Theresa Willett, BahareFarhadian, Michael Jeng, Margo Thienemann, and Jennifer Frankovich. "Hypoferritinemia and iron deficiency in youth with pediatric acute-onset neuropsychiatric syndrome." Pediatric Research (2020): 1-8.

20. Ishaq, Shahid, and EjazMazari. "MAJOR CLINICAL RISK FACTORS FOR FEBRILE SEIZURES IN CHILDREN AGED SIX TO SIXTY MONTHS." Professional Medical Journal 27, no. 5 (2020). 